hatching apparatus, only one showed straight radial pores in the base. Several had grown on a curve to a certain point, and then assumed a straight radial direction: but it is remarkable to note that the change was abrupt and not gradual. Part of one of these specimens (in which the centre was slightly damaged) is shown in Fig. $1 b$.

Finally a number of specimens of the typical form of this species from an outside pond were examined, and all had normal straight basal pores.

There seems to be a definite correlation between this change of orientation and the situation of the animal in a current of water of constant direction of flow. The specimen shown in Fig. $1 a$ seems from the orientation of the earliest visible part of its shell to have settled with its long axis along the tube, that is, in line with the current. This is consistent with the elongated shape of the larva. When examined it had swung through almost a right angle so as to lie across the current.

The following table shows the mean angular deviation from the direction of flow of the current, in relation to size, for 192 specimens from the edges of boards in the hatching ponds which were subject to an intermittent current of constant direction.

\begin{tabular}{|c|c|c|c|c|c|}
\hline Length in mm. .. & $0-1$ & $1-2$ & $2-3$ & $3-4$ & $4-5$ \\
\hline No. of specimens & 6 & 94 & 60 & 26 & 6 \\
\hline $\begin{array}{c}\text { Mean deviation } \\
\text { from current .. }\end{array}$ & $27^{\circ}$ & $49^{\circ}$ & $60^{\circ}$ & $75^{\circ}$ & $70^{\circ}$ \\
\hline
\end{tabular}

Some of these specimens were removed, and most showed curved growth in the base. A sample from the flat side of the boards where there was no current, showed no change of orientation in the larger individuals, and straight radial basal growth in all cases.

From these considerations it would appear that if a barnacle larva settles in a steady stream of water, it tends to attach itself so as to lie along the current, but immediately after metamorphosis it commences to rotate, and continues to do so until it lies across the current, in which position it then remains.

Marine Biological Station,

Hilary B. Moore.

Port Erin, I.O.M.

Nov. 10.

\section{Electromagnetic Mass}

Bx the modifications of Maxwell's field equations recently proposed ${ }^{1}$ it is possible to revive the old idea of the electromagnetic origin of inertia. The mass of the electron can then be calculated from its charge and the constants of the field equations (the velocity of light $c$, and the absolute field $a^{-1}$ ). It can be shown that the Lorentz equations for the motion of an electron in an external field are approximately true, and that the energy is given by $m c^{2}$. (The disagreement of these quantities stated in the Royal Society paper referred to above turned out to be a mistake.) The tensor $S$, the components of which are Maxwell's stresses, density of momentum and of energy, can be represented in two different forms, one using the Iaagrangian,

$$
L=a^{-2}\left\{1-\left[1+a^{2}\left(B^{2}-E^{2}\right)\right]^{\frac{1}{3}}\right\},
$$

the other the Hamiltonian,

$$
\mathrm{H}=a^{-2}\left\{\left[1+a^{2}\left(B^{2}-E^{2}\right)\right]^{-\frac{1}{2}}-1\right\},
$$

where $E$ is the electric and $B$ the magnetic field vector. For example, the 44-component of $S$, representing density of energy, is given by

$$
S_{44}=L+D E=-(\mathrm{H}+H B),
$$

where the vectors $H, D$ are connected with $B, E$ by

$$
H=\frac{\delta L}{\delta \bar{B}}, D=\frac{\delta L}{\delta E} \text { or } B=\frac{\delta \mathrm{H}}{\delta \bar{H}}, E=\frac{\delta \mathrm{H}}{\delta D} .
$$

For an electron at rest $(H=B=0)$ the mass $m$ is related to the total energy by the equation

$$
m c^{2}=\frac{1}{4 \pi} \int \mathrm{H} d V=\frac{2}{3} e \varphi(0)=1 \cdot 2361 \frac{e^{2}}{r_{0}},
$$

where $r_{0}=\sqrt{\overline{a e}}$ and

$\varphi(0)=\frac{e}{r_{0}} \int_{0}^{\infty} \frac{d x}{\sqrt{1+x^{4}}}=\frac{e}{r_{0}} \int_{0}^{\frac{\pi}{2}} \frac{d \alpha}{\sqrt{1-\frac{1}{2} \sin ^{2} \alpha}}=\frac{e}{r_{0}} 1 \cdot 8541 \ldots$

is the value of the potential at the centre of the electron. By integrating the conservation law for $S$, we obtain the Lorentz equations of motion for external fields which contain only those wave-lengths which are large compared with $r_{0}$.

From this it follows that Dirac's wave equation is the adequate expression for the quantum-mechanical laws of motion of the electron in external fields of this kind. Further, the mass appears here to be selfenergy which justifies the suppression of the corresponding terms occurring in the quantum mechanical laws of interaction of several particles.

MAX BORN.

L. INFELD.

\section{Hills Road,}

Cambridge.

Dec. 4.

${ }^{1}$ NAtURe, 132, 282, Aug. 19, 1933. Proc. Roy. Soc., In the press.

\section{Raman Spectrum of Heavy Water (By CABLE)}

The Raman spectrum of heavy water has been obtained by 2536 excitation of 8 c.c. of 18 per cent heavy water in a quartz tube $35 \mathrm{~cm}$. in length in contact with a quartz mercury vacuum tube. Two Raman bands were obtained with an intensity ratio of one to four, the new one having a mean wavelength of $2713 \mathrm{~A}$. due to water molecules containing one atom of heavy hydrogen. The frequency difference was 2577, against 3420 for ordinary water. Cross and Van Vleck (J. Chem. Phys., June) have calculated a Raman frequency difference of 2720 for heavy water vapour, but a lower value is to be expected for the liquid, which Dieke has calculated as agreeing with my value within four per cent. There appeared to be slight indication of the band due to molecules containing two atoms of heavy hydrogen, but the faint continuous background made any certain measurements impossible. The heavy water was prepared by John W. Murray, of the Department of Chemistry.

Johns Hopkins University,

R. W. Woon.

Baltimore.

Dec. 16. 